\title{
DISTRIBUCIÓN DEL GÉNERO HYALELLA SMITH, 1874 (CRUSTACEA: AMPHIPODA) EN EL SUR DE LA PATAGONIA Y TIERRA DEL FUEGO
}

\author{
DISTRIBUTION OF GENUS HYALELLA SMITH, 1874 (CRUSTACEA: AMPHIPODA) IN \\ SOUTHERN PATAGONIA AND TIERRA DEL FUEGO
}

Marcela Vega ${ }^{1,2}$, Patricio De los Ríos ${ }^{2}$ \& Andrés Mansilla ${ }^{3}$

Los anfípodos son un orden de crustáceos macroscópicos, muchos de ellos marinos y otros de agua dulce. Tienen diversos hábitos alimenticios pudiendo ser herbívoros, detritívoros, carnívoros u omnivoros (Väinölä et al. 2008). Esta situación implica que los anfípodos tengan un rol clave como descomponedores de materia orgánica, y como presas para aves, peces, y macroinvertebrados, por ejemplo insectos (Jara et al. 2006).

La distribución en Chile de especies de anfípodos de aguas continentales del género Hyalella, presentó en un comienzo una situación de profunda confusión taxonómica, lo que se aclaró con recientes estudios, en que se confirmó la existencia de siete especies para aguas continentales chilenas: $\mathrm{H}$. simplex, H. fossamancinii, H. kochi, H. chiloensis, $H$. costera, H. araucana y H. franciscae (González
\& Watling 2001, 2003, González 2003, Jara et al. 2006). El presente estudio consiste en una revisión de especies del género Hyalella para la zona sur de la Patagonia y de la Isla Grande de Tierra del Fuego de Argentina y Chile.

\section{Estatus taxonómico}

Phylum: Arthropoda Superclase: Crustacea Clase: Malacostraca Superorden: Perecarida Orden: Amphipoda Familia: Hyalellidae Hyalella araucana Grosso \& Peralta, 1999 Hyalella franciscae González \& Watling, 2003 Hyalella simplex Schellenberg, 1943

\footnotetext{
${ }^{1}$ Universidad de Chile, Facultad de Ciencias, Estudiante Programa de Magíster en Ciencias Biológicas, Casilla 653, Santiago de Chile, biomacriel@gmail.com

${ }^{2}$ Universidad Católica de Temuco, Facultad de Recursos Naturales, Escuela de Ciencias Ambientales, Casilla 15-D, Temuco, Chile.

${ }^{3}$ Universidad de Magallanes, Departamento de Ciencias y Recursos Naturales, Av. Bulnes 01855, Punta Arenas, Chile. Instituto de Ecología y Biodiversidad. Casilla 653, Santiago, Chile.
} 
Distribución geográfica (Fig.1)

Hyalella araucana Grosso \& Peralta 1999

\section{ARGENTINA}

Arroyo Calafate (50²0'S; $72^{\circ} 15^{\prime}$ W); Lago

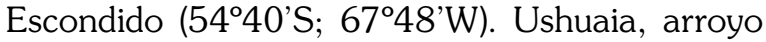
cercano a lago Escondido (54\% $43^{\prime}$ S- $\left.68^{\circ} 01^{\prime} \mathrm{W}\right)$. (Dos Santos et al. 2008)

\section{CHILE}

Lago Sarmiento $\left(51^{\circ} 04^{\prime} \mathrm{S}, 7^{\circ} 45^{\prime} \mathrm{W}\right)$; Río Seco (5306' S; 7053' W); Río de los Ciervos 5311' S, 7056' W)(González \& Wattling 2003); Humedal de Tres Puentes (53 06' S; 70 $52^{\prime} \mathrm{W}$ ), Laguna a la salida de Porvenir (5317' S; 70¹9' W)

Hyalella simplex Schellenberg, 1943 (González \& Watling 2003)

\section{CHILE}

Punta Delgada (72¹5' S; 72 45’ W)(González

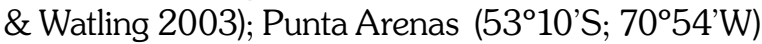
(González 2003); Laguna Redonda (5101' S; $72^{\circ} 52^{\prime}$

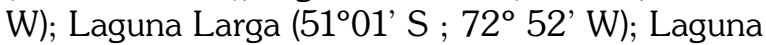
Monserrat (51 $07^{\prime} \mathrm{S}$; $72^{\circ} 57^{\prime} \mathrm{W}$ ); Puerto Natales (5142' S; 7247' W); Río Seco (5306' S; 7053' W); Tres Puentes (53 06' S; $70^{\circ} 52^{\prime} \mathrm{W}$ ); Porvenir (53ำ' S; 70²19' W).

\section{Hyalella franciscae González \& Watling 2003}

\section{CHILE}

Laguna El Paso, Torres del Paine (50²9'S-

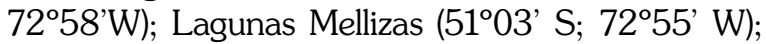
Laguna Redonda (51 $01^{\prime}$ S; $72^{\circ} 52^{\prime} \mathrm{W}$ ), Laguna Larga (5101' S; 72 52' W), (González \& Watling 2003)

De las siete especies del género Hyalella mencionadas para Chile, en el extremo sur son reportadas según bibliografía a: $H$. araucana, $H$. simplex y $H$. franciscae. Las tres especies habitan el agua dulce, ambientes epigeos, $H$. araucana y $H$. franciscae además se les puede encontrar en ambientes litorales. La distribución geográfica de $H$. araucana comprende la zona norte y sur de la Patagonia (Fig.1, González 2003, González \& Watling 2003), provincia de Santa Cruz en Argentina, y Tierra del Fuego, (Fig.1, Dos Santos et al. 2008). Por su parte Hyalella simplex y Hyalella franciscae se limita al extremo sur de Sudamérica, es- pecíficamente sólo en la región de Magallanes (Fig.1, González \& Watling 2003, González 2003).

Sobre la base de los resultados, se podría explicar que la presencia de especies en Argentina y Chile, como sucede con $H$. araucana, se debería probablemente a que la cordillera de los Andes tiende a desaparecer en la zona centro y sur de la Patagonia lo cual favorecería la dispersión de especies en esta región (Morrone 2004, 2006). Similares resultados han sido observados en estudios panbiogeográficos con copépodos calanoideos (Menu-Marque et al. 2000), donde se tiende a observar una amplia dispersión de especies en la zona centro y sur de la Patagonia de Argentina y Chile, situación que no sucedería en latitudes correspondientes al norte de la Patagonia (> $41^{\circ}$ S ) ( Morrone 2004, 2006 ).

No obstante, se requiere más información para entender patrones biogeográficos y de distribución geográfica, pues dentro de los pocos datos, son muy escasos para la región de Aisén, si bien la literatura indica la presencia de $H$. franciscae para el lago Risopatrón $\left(44^{\circ} 15^{\prime} \mathrm{S} ; 7^{\circ} 31^{\prime} \mathrm{W}\right)$ y $\mathrm{H}$. chiloensis para el río Nirepan (453' S; 72 ${ }^{\circ}$ 04' W). De ambas especies, $H$. franciscae estaría distribuida en las regiones de Aisén y Magallanes (44-51 $\left.{ }^{\circ} \mathrm{S}\right)$, mientras que $H$. chiloensis estaría entre los 36 y $45^{\circ} \mathrm{S}$ (González 2003). Considerando los antecedentes de la literatura y los del presente estudio, probablemente la región de Aisén sería una zona de transición entre especies del territorio de Chile central y de la Patagonia, por lo que se requeriría de más estudios biogeográficos y ecológicos.

\section{AGRADECIMIENTOS}

El presente estudio fue financiado por el proyecto DGI- CDA-01-07 de la Dirección de Investigación y Postgrado de la Universidad Católica de Temuco. De igual modo, se agradece el apoyo de la Escuela de Ciencias Ambientales de la Universidad Católica de Temuco.

\section{LITERATURA CITADA}

Dos Santos, A. L. F., P. B. Araujo \& G. Bond-Buckup 2008. New species and new reports of Hyalella (Crustacea, Amphipoda, Dogielinotidae) from Argentina. Zootaxa 1760: 24-36.

González, E. R., G. Bon-Buckup \& P. B. Araujo 2006. Two new species of Hyalella from southern Brazil (Amphipoda: Hyalellidae) with a taxonomic key. 
Journal of Crustacean Biology 26 (3): 355-365.

González, E. R \& L. Watling 2003. A new species of Hyalella from the Patagonia, Chile with redescription of H. simplex Schellenberg, 1943 (Crustacea: Amphipoda). Journal of Natural History 37(17): 2077-2094.

González, E. R., 2003. The freshwather amphipods Hyalella Smith, 1874 in Chile (Crustacea: Amphipoda). Revista Chilena de Historia Natural 76: 623-637.

González, E. R. \& L. Watling 2001. Three new species of Hyalella from Chile (Crustacea: Amphipoda: Hyalellidae). Hidrobiologia 464: 175-199.

Jara, C. G., E. H. Rudolph \& E. R. González 2006. Estado de conocimiento de los malacostráceos dulceacuícolas de Chile. Gayana 70 (1): 40-49. Menu-Marque, S., J. J. Morrone \& S. Locascio de
Mitrovich 2000. Distributional patterns of South American species of Boeckella (Copepoda, Centropagidae): a track analysis. Journal of Crustacean Biology 20: 262-272.

Morrone, J.J. 2004. Panbiogeografía, componentes bióticos y zonas de transición. Revista Brasileira de Entomologia 48: 149-162.

Morrone, J. J. 2006. Biogeographic areas and transition zones of Latin American and the Caribbean islands based on panbiogeographic and cladistic analyses of the entomofauna. Annual Review of Entomology 51: 467-494.

Väinölä, R., J. D. S. Witt, M. Grabowski, J. H. Bradbury, K. Jazdzewski \& B. Sket 2008. Global diversity of amphipods (Amphipoda; Crustacea) in freshwater. Hidrobiologia 595: 241-255.

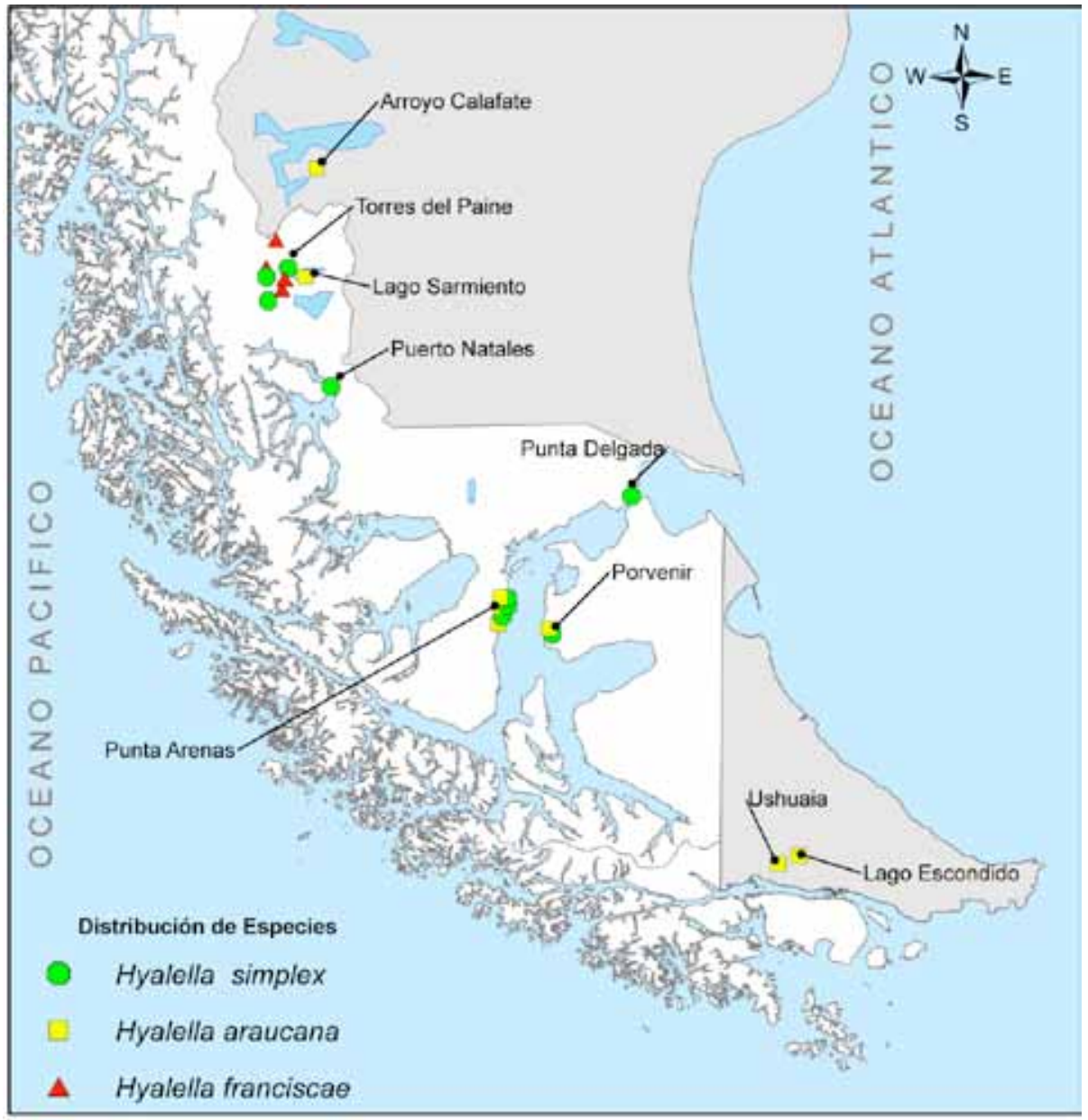

Fig.1. Mapa con la localización de las especies mencionadas en el presente estudio. 